nisationen wie Vaterländische Frauenvereine gegründet, um die Pflege der Kranken zu übernehmen und die Opfer kriegerischer Auseinandersetzung sowie wachsender sozialer Missstände zu versorgen. Diese schlossen sich rasch der Rotkreuzbewegung an, oftmals gingen aus ihnen DRK-Schwesternschaften hervor. Der Beruf einer Krankenpflegerin bot alleinstehenden, wirtschaftlich nicht abgesicherten Frauen eine berufliche Chance und eine neue Lebensperspektive. Ein Eintritt in eine DRK-Schwesternschaft war damals auch ein Eintritt in eine Fürsorgegemeinschaft, die sich im Laufe der Jahre in eine Verantwortungsgemeinschaft gewandelt hat.

\section{Berufliche Perspektiven}

Die Vielfalt der beruflichen Perspektiven, die DRK-Schwesternschaften ihren Mitgliedern bieten können, ist eng verzahnt mit einer systematischen Fort-

\section{ZAHLEN_DATEN_FAKTEN}

Mitwirkung, Mitbestimmung und Mitverantwortung - das Engagement der Mitglieder ist gefragt.

_ Mitglieder im VdS: 33 DRK-Schwesternschaften bundesweit

_ Vorstand: Generaloberin Gabriele Müller-Stutzer (Präsidentin) Generaloberin Edith Dürr (Vizepräsidentin), Klaus Zimmermann (Vizepräsident)

_ 22.000 Mitglieder der DRK-Schwesternschaften: Gesundheits- und Krankenpflegerinnen, Gesundheits- und Kinderkrankenpflegerinnen, Altenpflegerinnen. Sie sind Teil eines deutschlandweiten Frauen-Netzwerkes und gehören der größten humanitären Organisation der Welt an

_ Zugehörigkeit: Mitgliedsverband im DRK e.V.

www.rotkreuzschwestern.de; Carstennstraße 58-60, 12205 Berlin vom DRK e.v. und Weiterbildung. Das reicht von einer fundierten Ausbildung auf hohem Niveau über qualifizierte Fort- und Weiterbildung - zum Teil in eigenen Fort- und
Weiterbildungsinstituten - bis hin zur Begleitung der akademischen Ausbildung. Talent und persönliche Wünsche stehen dabei im Vordergrund.

\title{
Nachgefragt
}

... bei Generaloberin Gabriele Müller-Stutzer, der Präsidentin des VdS. Wir wollten unter anderem wissen, welche Themen für ihren Verband derzeit im Mittelpunkt stehen.

\section{? Frau Generaloberin Müller-Stutzer,} was zeichnet den VdS aus?

Müller-Stutzer: Der VdS wurde 1882 gegründet und gehört zum ältesten Teil der Rotkreuzbewegung. Unser berufliches Handeln wird durch die Berufsethischen Grundsätze der DRK-Schwesternschaften bestimmt. Wir sind als Mitgliedsverband des DRK aktiv in einem Spitzenverband der freien Wohlfahrtspflege und setzen uns für die Professionalisierung der Pflege ein, indem wir jährlich ca. 4.000 Schülerinnen und Schüler an 69 Pflegeschulen ausbilden. Von Anfang an ist uns die Absicherung unserer Rotkreuzschwestern auch im Alter - wichtig. Als Interessenvertreter arbeiten wir eng mit dem DRKBundesverband und anderen Pflege- und Gesundheitsverbänden zusammen. Zudem sind wir die einzige konfessionell nicht gebundene Schwesternschaft, das heißt Religionszugehörigkeit der Mitglieder spielt bei uns keine Rolle.
? Welche drei Themen stehen für Ihren Verband derzeit im Mittelpunkt? Müller-Stutzer: Zum einen die Verbesserung der Rahmenbedingungen für professionelle Pflegekräfte. Dazu gehört, dass wir uns stark machen für die Errichtung von Pflegekammern und ein klares Votum für eine generalistische Ausbildung abgeben.

? Stichpunkt Generalistik. Wie steht der VdS dazu?

Müller-Stutzer: Wir sprechen uns seit Langem für eine generalistische Pflegeausbildung aus. Denn nur mit dieser Ausbildung, die übergreifende Qualifikationen aus allen drei Bereichen der Pflege sowie ein am gesamten Lebensverlauf orientiertes Verständnis von Krankheit und Pflegebedürftigkeit vermittelt, kann den sich verändernden gesellschaftlichen, berufsspezifischen und arbeitsorganisatorischen Anforderungen an die professio-

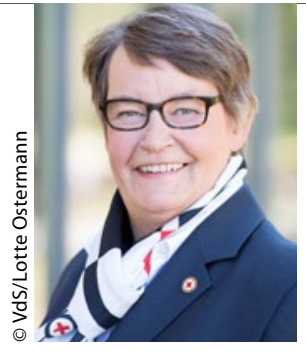

Gabriele Müller-Stutzer

Präsidentin des VdS

nell Pflegenden Rechnung getragen werden.

? Zur Professionalisierung der Pflege gehört ja auch die Aus-, Fort- und Weiterbildung. Hat der VdS konkrete Vorstellungen dazu?

Müller-Stutzer: Wir kümmern uns um unseren Nachwuchs, indem wir Ausbilder und Qualifizierer sind. Als ein Trägerverband des Deutschen Bildungsrates arbeiten wir mit an Richtlinien für die Aus-, Fort- und Weiterbildung. Hier sind die Verbände aber vor dem Hintergrund des Pflegeberufsgesetzes auch aufgefordert, sich für bundesrepublikanisch einheitliche Regelungen zur Krankenpflegehilfe- und Altenpflegehilfe-Ausbildung einzusetzen.. 